\title{
Phenotype of CM-AVM2 caused by variants in EPHB4: how much overlap with hereditary hemorrhagic telangiectasia ( $\mathrm{HHT})$ ?
}

\author{
Whitney L. Wooderchak-Donahue, PhD ${ }^{1,2}$, Gulsen Akay, MD ${ }^{1,3}$, Kevin Whitehead, MD ${ }^{4}$, Eric Briggs ${ }^{1}$, \\ David A. Stevenson, $\mathrm{MD}^{5}$, Brendan $\mathrm{O}^{\prime}$ Fallon, $\mathrm{PhD}^{1}$, Matthew Velinder, $\mathrm{PhD}^{6}$, Andrew Farrell, $\mathrm{PhD}^{6}$, \\ Wei Shen, $\mathrm{PhD}^{1}$, Emma Bedoukian, MS ${ }^{7}$, Cara M. Skrabann, MD ${ }^{7,8}$, Richard J. Antaya, MD ${ }^{9}$, \\ Kate Henderson, $\mathrm{MS}^{10}$, Jeffrey Pollak, MD ${ }^{10}$, James Treat, MD ${ }^{11}$, Ronald Day, MD ${ }^{12}$, \\ Joseph E. Jacher, $\mathrm{MS}^{13}$, Mark Hannibal, MD ${ }^{13}$, Kelly Bontempo, MS ${ }^{14}$, Gabor Marth, PhD ${ }^{6}$, \\ Pinar Bayrak-Toydemir, MD, PhD ${ }^{1,2}$ and Jamie McDonald, $\mathrm{MS}^{2,15}$
}

\begin{abstract}
Purpose: EPHB4 variants were recently reported to cause capillary malformation-arteriovenous malformation 2 (CM-AVM2). CMAVM2 mimics RASA1-related CM-AVM1 and hereditary hemorrhagic telangiectasia (HHT), as clinical features include capillary malformations (CMs), telangiectasia, and arteriovenous malformations (AVMs). Epistaxis, another clinical feature that overlaps with HHT, was reported in several cases. Based on the clinical overlap of CM-AVM2 and HHT, we hypothesized that patients considered clinically suspicious for HHT with no variant detected in an HHT gene (ENG, ACVRL1, or SMAD4) may have an EPHB4 variant.
\end{abstract}

Methods: Exome sequencing or a next-generation sequencing panel including EPHB4 was performed on individuals with previously negative molecular genetic testing for the HHT genes and/or RASA1.

Results: An $E P H B 4$ variant was identified in ten unrelated cases. Seven cases had a pathogenic EPHB4 variant, including one with mosaicism. Three cases had an EPHB4 variant of uncertain significance. The majority had epistaxis (6/10 cases) and telangiectasia (8/10 cases), as well as CMs. Two of ten cases had a central nervous system AVM.

Conclusions: Our results emphasize the importance of considering CM-AVM2 as part of the clinical differential for HHT and other vascular malformation syndromes. Yet, these cases highlight significant differences in the cutaneous presentations of CM-AVM2 versus HHT.

Genetics in Medicine (2019) 21:2007-2014; https://doi.org/10.1038/s41436019-0443-z

Keywords: capillary malformation; CM-AVM; EPHB4; HHT; telangiectasia

\section{INTRODUCTION}

Capillary malformation-arteriovenous malformation (CMAVM) syndrome (OMIM 608354) is an autosomal dominant disorder, the only identified gene for which was Ras p21 protein activator 1 (RASA1, chromosome 5q14) (ref. ${ }^{1}$ ) until the recent report by Amyere and colleagues of variants in the Ephrin B4 (EPHB4, chromosome 7q22) gene in patients with the disorder. ${ }^{2}$ They proposed that the disorders caused by RASA1 and EPHB4 be called CM-AVM1 and CM-AVM2, respectively. In another subsequent study, an EPHB4 variant was found in 5 of 51 patients with a vein of Galen aneurysmal malformation, only two of which were observed to have cutaneous CMs. $^{3}$

RASA1-related CM-AVM1 is characterized by hereditary multifocal cutaneous CMs, with or without AVMs/arteriovenous fistulas (AVFs), and Parkes Weber syndrome (multiple micro-AVFs and soft- and skeletal-tissue hypertrophy of the affected limb). CMs characteristic of this syndrome are described as atypical in that they are smaller (variable, but often $1-2 \mathrm{~cm}$ in diameter) and more pink versus red as

\footnotetext{
${ }^{1}$ ARUP Institute for Clinical and Experimental Pathology, Salt Lake City, UT, USA; ${ }^{2}$ Department of Pathology, University of Utah, Salt Lake City, UT, USA; ${ }^{3}$ Zeynep Kamil Training and Research Hospital, Istanbul, Turkey; ${ }^{4}$ Division of Cardiovascular Medicine, Department of Medicine, HHT Center, University of Utah, Salt Lake City, UT, USA; ${ }^{5}$ Division of Medical Genetics, Department of Pediatrics, Stanford University, Stanford, CA, USA; ${ }^{6}$ USTAR Center for Genetic Discovery, University of Utah, Salt Lake City, UT, USA; ${ }^{7}$ Roberts Individualized Medical Genetics Center, Children's Hospital of Philadelphia, Philadelphia, PA, USA; ${ }^{8}$ Division of Human Genetics, Department of Pediatrics, Perelman School of Medicine, University of Pennsylvania, Philadelphia, PA, USA; ${ }^{9}$ Departments of Dermatology and Pediatrics and HHT Program, Yale University School of Medicine, New Haven, CT, USA $;{ }^{10}$ Department of Radiology and Biomedical Imaging, and HHT Program, Yale University School of Medicine, New Haven, CT, USA; ${ }^{11}$ Dematology Section, Department of Pediatrics, Children's Hospital of Philadelphia, Philadelphia, PA, USA $;{ }^{12}$ Division of Pediatric Cardiology, Department of Pediatrics, University of Utah, Salt Lake City, UT, USA; ${ }^{13}$ Department of Pediatrics and Communicable Diseases, Division of Pediatric Genetics, Metabolism \&Genomic Medicine, University of Michigan, Ann Arbor, MI, USA; ${ }^{14}$ Advocate Children's Hospital, Park Ridge, IL, USA; ${ }^{15}$ Department of Radiology, HHT Center, University of Utah, Salt Lake City, UT, USA. Correspondence: Jamie McDonald (Jamie. mcdonald@hsc.utah.edu)

These authors contributed equally: Whitney L. Wooderchak-Donahue and Gulsen Akay
} 
compared with the "typical" CMs, also known as "port wine stains." They are randomly distributed over the body, and many have a surrounding white halo, which represents microAVM shunting/vascular steal. Approximately half of AVMs/ AVFs found in cases with CM-AVM1 are located in the central nervous system. ${ }^{4,5}$ RASA1-encoded p120RASGAP is a direct effector of EPHB4, implicating the EPHB4-RAS-ERK signaling pathway as a major cause for AVMs in these disorders. ${ }^{2}$ Molecular diagnostic testing is useful in the diagnosis and management of these cases. ${ }^{6,7}$ However, many cases suspected to have CM-AVM do not have an identifiable variant in the RASA1 gene (ranging from $22 \%$ to $79 \%$ depending on the study cohort). ${ }^{5-7}$

Based on a study of 110 individuals from 54 families in whom an EPHB4 variant was detected, Amyere and colleagues described CM-AVM2 as also characterized by pink to red cutaneous CMs, most often on the extremities and trunk with $\sim 25 \%$ surrounded by a white halo. An AVM/AVF was present in $18 \%$ of cases (vein of Galen aneurysmal malformations $[n=2]$ and intraspinal AVF $[n=1]$, face $[n=8]$, and the extremities $[n=1])$. Eight vascular malformations described as "Parkes Weber lesions" were reported on extremities. Bier spots were noted in $12 \%$ of cases, and telangiectases, especially of the upper thorax, lips, and periorally, in $15 \%$ of cases. The CM-AVM2 phenotype was said to mimic CM-AVM1 syndrome caused by variants in RASA1, but also hereditary hemorrhagic telangiectasia (HHT) due to the occurrence of telangiectases, central nervous system AVMs, and a few cases noted to have recurrent epistaxis. ${ }^{2}$

HHT, another autosomal dominant vascular malformation disorder, is caused by variants in one of three transforming growth factor beta (TGF- $\beta$ ) signaling pathway genes (ENG, ACVRL1, and SMAD4) (ref. ${ }^{8}$ ). HHT is characterized by (1) recurrent epistaxis; (2) telangiectases of the lips, oral cavity, fingers, nasal mucosa, and gastrointestinal tract; and (3) internal organ AVMs (particularly in the lungs, liver, and brain) according to the Curaçao criteria. ${ }^{9,10}$ Epistaxis and telangiectases exhibit age-dependent onset and progression. Most patients report the appearance of telangiectases during the third decade, they are almost never "diffuse" or "too many to count," and are rarely found on the trunk or extremities other than hands. ${ }^{10,11}$ Variants in the bone morphogenetic 9 (BMP9) gene GDF2 were reported in three unrelated individuals considered clinically suspicious for HHT. ${ }^{12}$ This disorder, since specified as HHT type 5 by OMIM (OMIM \#615506), was reported in «1\% of individuals referred for molecular genetic testing for $\mathrm{HHT}^{12}$. Clinical findings in these three cases were noted to differ from those of HHT as defined by the Curaçao criteria, and to possibly overlap with CMAVM. ${ }^{9,12}$

Pathogenic RASA1 variants were recently reported in individuals clinically suspected to have HHT. ${ }^{7,13}$ Considering this and the 2017 report by Amyere and colleagues of the clinical overlap between CM-AVM2 and HHT, we hypothesized that individuals previously negative for a mutation in the HHT genes may have a causative EPHB4 variant. We report sequencing results for the $E P H B 4$ gene in individuals with previous negative testing for ENG, ACVRL1, SMAD4, and in some cases GDF2 or RASA1. These results will help define the clinical overlap between CM-AVM2 and HHT, and whether EPHB4 should be included in HHT gene panel testing to increase clinical sensitivity.

\section{MATERIALS AND METHODS}

\section{Subjects}

Subjects were unrelated individuals who had tested negative for a variant in an HHT gene (ENG, ACVRL1, SMAD4), plus/ minus RASA1 and GDF2. These individuals represented a broad spectrum regarding manifestations suggestive of a vascular malformation syndrome, and also in regard to their clinical evaluation for telangiectases/AVMs. A minority $(\sim 25 \%)$ met consensus clinical diagnostic criteria for HHT. Most had either one or two reported manifestations of HHT. Some were considered suspicious for both HHT and CMAVM. This study was approved by the University of Utah Institutional Review Board (IRB 31050, 35637, 39582, and 20480). Written consent from each patient/parent was obtained for the use of photographs.

\section{Next-generation sequencing (NGS) and data analysis}

Exome sequencing was performed on an initial 27 cases, and an NGS panel for an additional 86 cases. Genomic DNA was extracted from peripheral blood using a Gentra Puregene Blood Kit (Qiagen, Valencia, CA). DNA was sheared, and Illumina adapters were added using SureSelect XT kit reagents (Agilent Technologies, Santa Clara, CA). Adapter-ligated DNA underwent hybridization with the biotinylated RNA baits designed to target either the whole exome or a small custom HHT panel for 24 hours at $65^{\circ} \mathrm{C}$. For the custom HHT panel, 120 nucleotide RNA baits were designed to specifically target the coding regions and exon/intron boundaries of ACVRL1, ENG, SMAD4, GDF2, RASA1, and EPHB4. Hybridized DNA targets of interest were captured using streptavidin-coated magnetic beads and eluted after a series of washes. Samples were pooled and sequenced on a HiSeq2500 instrument (Illumina, San Diego, CA) using $2 \times$ 100 paired-end reads. Exome and panel samples were sequenced to an average depth of $\sim 100 \times$ and $\sim 250 \times$ coverage, respectively.

Sequences were aligned to the human genome reference (hg19) sequence using the Burrows-Wheeler Alignment tool (BWA 0.5.9) with default parameters. ${ }^{14}$ Polymerase chain reaction (PCR) duplicates were removed using the SAMtools package, ${ }^{15}$ and base quality score recalibration, local realignment, and variant calling were performed using the Genome Analysis Toolkit (GATK v1.3) (ref. ${ }^{16}$ ).

\section{Sanger sequencing}

Potential pathogenic variants were confirmed using Sanger sequencing. DNA from additional sample types from a case with mosaicism were evaluated using Sanger sequencing. Primer sequences are available upon request. Amplicon 
fragments were bidirectionally sequenced and sequences were compared with the reference sequence NM_004444.4 using Mutation Surveyor (SoftGenetics, State College, PA, USA).

\section{RESULTS}

Ten unrelated individuals (from a group of $>100$ unrelated patients with widely variable clinical findings that had raised suspicion for a telangiectasia/AVM syndrome) were identified to have a variant in the EPHB4 gene (Table 1). Seven cases had a pathogenic EPHB4 variant that caused loss of function (Supplemental Fig. 1); six of the seven were novel. One case (case 7) had a mosaic EPHB4 variant at 19\%, the first report of mosaicism as a cause of CM-AVM2. Three cases had an $E P H B 4$ variant of uncertain significance that was predicted to be damaging by multiple prediction algorithms. The majority of cases had epistaxis (6/10 cases) and telangiectasia (8/10 cases), in addition to CMs. Two had a central nervous system AVM. A family history was reported in four cases, although family members were not examined in most cases (Table 1). Parents were tested confirming de novo variants in two cases (cases 5 and 7) not known to have a family history.

Case 1: 10-year-old with 2-3 nosebleeds/month of 6-15 minutes duration. This individual had approximately 45 cutaneous vascular lesions; 15 were $0.5-2 \mathrm{~cm}$ pink CMs, and 30 were pinpoint to pinhead size telangiectases. Four CMs and 16 telangiectases were noted on the hands bilaterally. On the arms, three CMs and one telangiectasia were noted; on the legs, four CMs and a cluster of approximately 12 telangiectases were noted. One CM was noted on the left shoulder, upper back, upper chest, and base of the neck. Some, but not the majority, had a surrounding halo (Fig. 1a, b). Bier spots were noted on the left forearm. A brain magnetic resonance image (MRI) was negative.

Case 2: 12-year-old with dermal lesions described as telangiectasia on the malar region and upper arms bilaterally. Recurrent nosebleeds with chronic sinusitis for one year at six years of age were reported, but had since resolved.

Case 3: 11-year-old with cutaneous telangiectases on the lips and periorally (Fig. 1c), left arm, right forehead, dorsum of hands, upper back, mucous opening to nose, and anterior tongue (too numerous to count). CMs were noted on the left arm (Fig. 1d) and back. The patient had several nosebleeds per year. Brain and spinal MRIs were negative.

Case 4: 13-year-old with telangiectases on the hands, lips, arms, legs, over the collarbone area, and a "birthmark" size spot on the wrist. Epistaxis of unknown frequency was reported. A brain MRI was negative. A brother was reported to have had a ruptured brain AVM.

Case 5: 17-year-old with CMs on the neck (4) (Fig. 1e), upper chest (1), left forearm (2) (Fig. 1f), left wrist (1), palmar right hand (1), and right shoulder (1). Prominent Bier spots were present on the arms (Fig. 1g) and legs bilaterally. No lesions were noted on the oral mucosa, lips, or face. No telangiectases were noted. A vein of Galen malformation was discovered in late gestation, with high output heart failure at birth. Parents and two siblings were examined with no significant findings noted. The individual was suspected to have CM-AVM but had previously tested negative for a RASA1 variant. Exome sequence revealed an EPHB4 variant (c.1423-6G >A, p.Gly475Thrfs ${ }^{\star} 39$ ) previously reported in a CM-AVM2 patient in which messenger RNA (mRNA) studies confirmed the splicing defect of an insertion of $4 \mathrm{bp}$ of intronic sequence resulting in a protein frameshift. ${ }^{2}$ This variant was absent in the patient's parents.

Case 6: 11 years of age at initial presentation, but when examined at 14 years of age had numerous telangiectases across the malar region (Fig. 1h), nose, philtrum, upper and lower lips (Fig. 1i), and upper chin, with fewer on her forehead and on the sides of her face and ears. Greater than 200 telangiectases were noted on the lower arms and hands bilaterally, some of which had halos. Capillary stains/ malformations were noted on the left temporal hair margin (Fig. 1j), left preauricular area near the mandible, right forearm, and upper back, ranging from $4 \times 6 \mathrm{~mm}$ to $20 \times 10$ $\mathrm{mm}$. The patient had no nosebleeds in the previous 12 months, but from ages 2 to 5 years had approximately six per year that lasted 20 minutes. A brain MRI was negative. The patient's father had scattered telangiectases on his face and capillary stains/malformations on the right hand $(22 \times 12$ $\mathrm{mm})$, right middle back $(22 \times 17 \mathrm{~mm})$, and a clear white capillary stain on the right upper shoulder $(38-27 \mathrm{~mm})$. He has 6-10 nosebleeds per year that last about 20 minutes. An 8year-old sister was reported to have 6-10 nosebleeds per year that last 20 minutes and multiple telangiectases on the upper lip to the vermilion border. Capillary stains/malformations were noted on the sister's left temporal region $(5 \times 2 \mathrm{~mm})$, two on the left jaw $(2 \times 1 \mathrm{~mm}$ and $2-3 \mathrm{~mm})$, under her nose (1-2 $\mathrm{mm})$, and about eight on her left hand that ranged from pinpoint to $2 \mathrm{~mm}$ in size, and left thumb $(4 \times 3 \mathrm{~mm})$. Unfortunately, familial samples were unavailable for testing.

\section{Mosaicism of EPHB4 causes CM-AVM2}

Case 7: 9-year-old with epistaxis of onset at age 1 year. Nosebleeds typically last 1-5 minutes and occur several times per week. Diffuse ("100s") telangiectases were noted on the hands (Fig. 1k), feet, arms, legs, back, abdomen, neck, face, palate, lips, and periorally. Telangiectases were individual in places, but in "patches" on many areas of the skin. Some lesions, particularly on the lower extremities, had a halo. A lesion, now a $2-3 \mathrm{~cm} \mathrm{CM}$, was first noticed at two months of age on the upper arm. CMs were noted on the arm (Fig. 11), neck (Fig. 1m), leg, and back. It was described as difficult to find a place on the body without lesions. MRIs of both the brain and cervical spine were negative. A novel mosaic EPHB4 variant was detected at $19 \%(158 / 851 \times$ coverage $)$ using an NGS panel and confirmed by Sanger sequencing (Fig. 2). The variant was not present in the patient's mother or father and was predicted to be deleterious by both SIFT and MutationTaster. ${ }^{17,18}$ In addition to DNA from peripheral blood, DNA extracted from saliva, buccal, and hair bulb samples also revealed mosaicism for this variant at $25 \%, 20 \%$, and $0 \%$, respectively. 


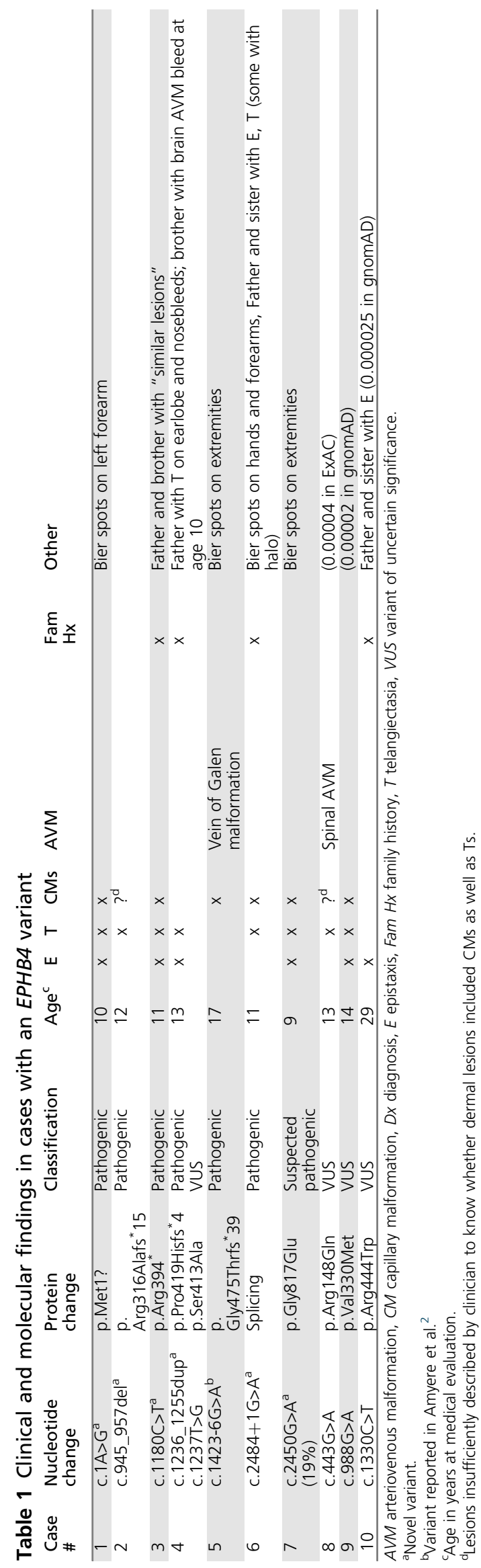

EPHB4 variants of uncertain significance identified in cases suspected to have HHT

Three additional cases had an EPHB4 variant of uncertain significance (VUS) (Table 1). Each was predicted to be damaging by multiple prediction algorithms (SIFT, ${ }^{17}$ PolyPhen, ${ }^{19}$ and MutationTaster ${ }^{18}$ ). All three variants had previously been identified in the ExAC or gnomAD databases at a very rare population frequency (ranging from 0.00002 to $0.00004)$.

Case 8: 13-year-old with telangiectases concentrated on the right upper chest, and an AVM of the cervicothoracic spine and right upper back. Although none of the dermal lesions were identified as being CMs, the clinical differential diagnosis was considered to include both HHT and CMAVM. It is thus likely that at least some of the lesions were more typical of CMs than telangiectases.

Case 9: 14-year-old with daily epistaxis (onset 11 years old), often "pouring" and difficult to stop, resulting in anemia. Telangiectases were noted on the lower neck/upper chest $(\sim 6)$ (Fig. 1n), face ( 6), lip (1) (Fig. 1o, arrow), arms (Fig. 1p), and hands bilaterally $(\sim 15)$. A small number of lesions resembled 1-2 mm sized punctate telangiectases (Fig. 1o) or 1-3 cm CMs (Fig. 1n), but the majority of the lesions were several-millimeter spidery telangiectases (Fig. 1p). A brain MRI was normal.

Case 10: 29-year-old with severe epistaxis (onset age 4-5 years) and severe idiopathic pulmonary arterial hypertension (onset less than 6 months of age), which raised suspicion of HHT. Although the patient had transposition of the great vessels that was palliated by surgery, there was not a hemodynamic reason this should cause pulmonary hypertension. No cutaneous vascular lesions were noted. The patient died of heart failure secondary to pulmonary hypertension and severe thrombocytopenia at age 29 years. The proband's 66-year-old father reported nosebleeds 3-4 times/month since early childhood that had required cautery and packing. $\mathrm{He}$ had one telangiectases on his left forearm. A 37-year-old sister reported two nosebleeds of 6-15 minutes duration monthly with an almost daily minor nosebleeds. A blanchable pink lesion ( $\sim 3 \mathrm{~mm}$ in size) was noted below her lip (Fig. 1q). A 41-year-old sister reported 2-3 nosebleeds per year lasting $<1$ minute. The EPHB4 variant detected in the proband was also detected in the father and above-described two sisters.

\section{DISCUSSION}

We present here a cohort of patients with EPHB4 variants ascertained from a heterogeneous group of patients with variable clinical manifestations suggestive of a possible telangiectasia syndrome. The majority had epistaxis $(6 / 10)$ and telangiectasia $(8 / 10)$, which are the hallmarks of HHT, in addition to CMs considered to be the classic cutaneous lesion of CM-AVM. A significant number of all telangiectases and CMs had halos, and four of ten cases were reported to have Bier spots, neither of which are characteristic of HHT. Two of ten cases (20\%) had a central nervous system AVM/AVF, and a brother of one case (case 4) was reported to have a brain 

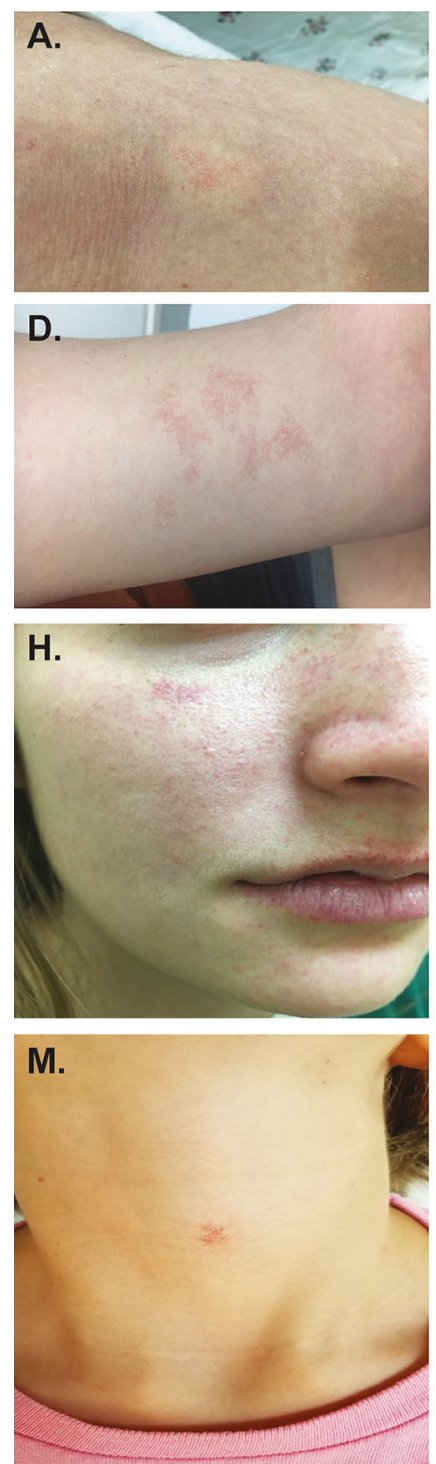
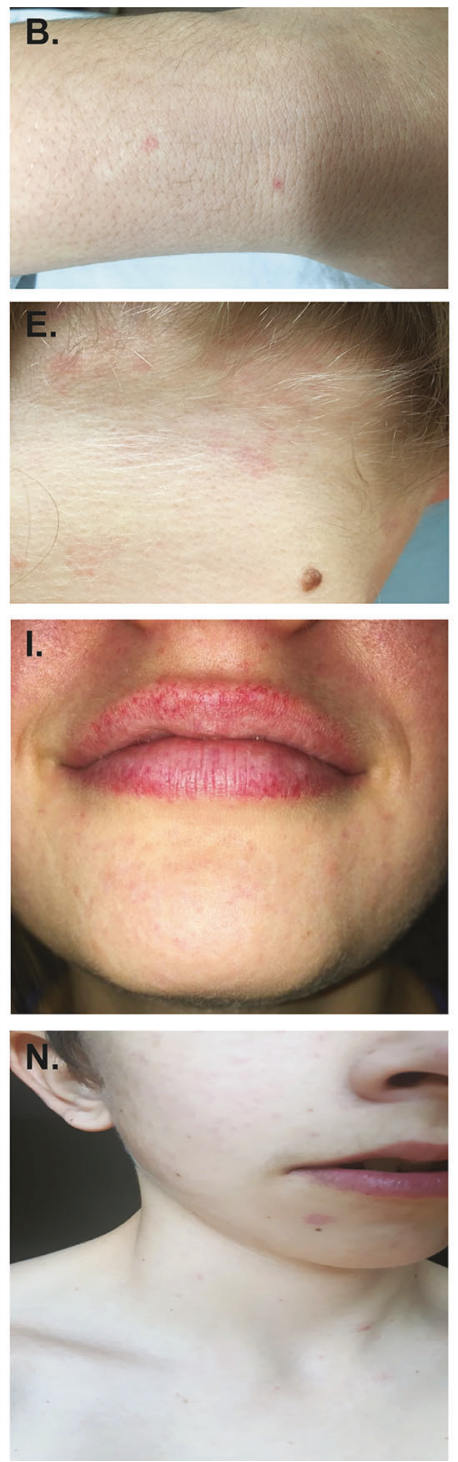

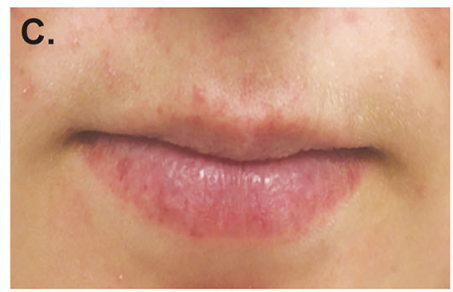

F.
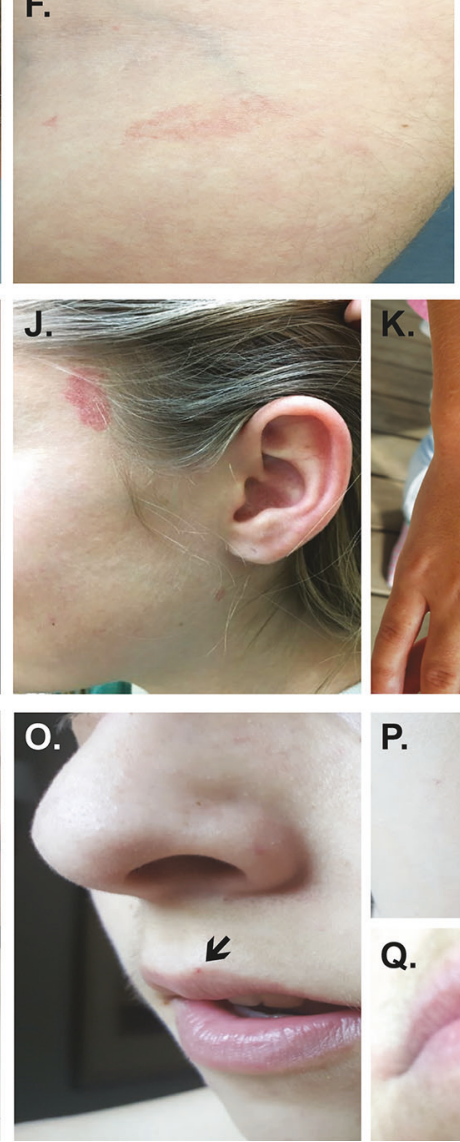
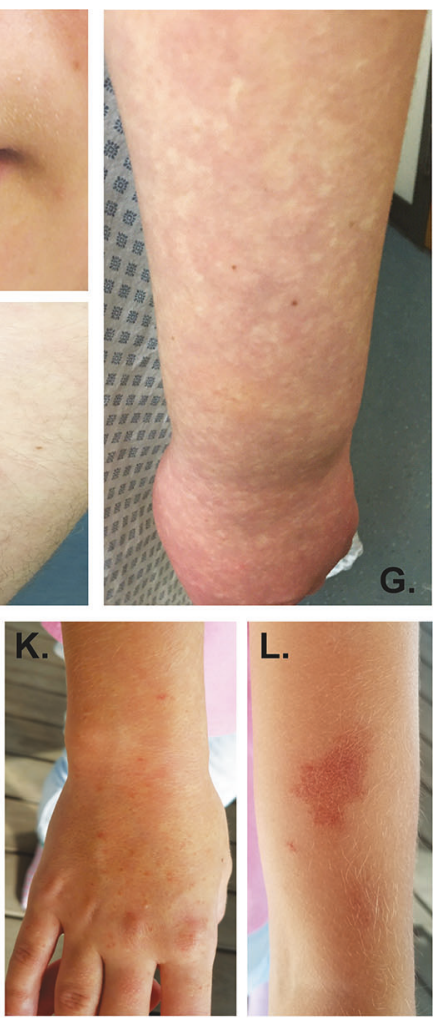

P.

Q.

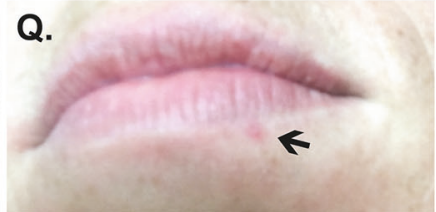

Fig. 1 Cutaneous findings in this series of capillary malformation--arteriovenous malformation 2 (CM-AVM2) patients. CM/telangiectases with surrounding halos below the right knee (a) and on the left wrist (b) from case 1. Telangiectases on lips (c) and CMs on the left arm (d) from case 3. CMs on the neck (e) and left forearm (f), and Bier spots on the left arm (g) from case 5. Numerous telangiectases across the malar region (h), nose (h), upper and lower lips (i), upper chin (i), and a capillary malformation on the left temporal hair margin (j) of case 6. Telangiectases on the arm and hand (k), and CMs on the $\operatorname{arm}(\mathbf{I})$ and neck $(\mathbf{m})$ of case 7. CM/telangiectases of variable sizes below the lower lip, and on the neck and chest (n) of case 9 . Telangiectasia on the lip (o, indicated with an arrow) and spidery telangiectases on the arm (p) of case 9. Small CM versus large telangiectasia below lip (q, indicated with an arrow) of the affected sister of case 10.

AVM that ruptured. This is compared with $3 / 110$ cases (2.7\%) with an EPHB4 variant previously reported, ${ }^{2}$ and $(\sim 10 \%)$ in HHT patients. ${ }^{11}$ Seven of ten patients in our series had an MRI of the spine and/or brain. This relatively high rate of screening may in part explain the higher rate of central nervous system AVM in our series than reported by Amyere et al. Central nervous system AVMs are considered characteristic findings in both CM-AVM and HHT, while AVMs in other solid organs (in particular lungs and liver) are considered characteristic only of HHT. Patients in this group considered suspicious for HHT in most cases, but not confirmed, generally have not had screening for asymptomatic AVMs other than in the brain.
One individual (case 10) was considered clinically suspicious for HHT based on epistaxis and idiopathic pulmonary arterial hypertension (PAH), a rare manifestation of HHT but not reported to date in CM-AVM. Additional reports of PAH in individuals with pathogenic variants in EPHB4 are needed to confirm the suspicion this raises that PAH may be a rare manifestation of CM-AVM2.

The first case series of patients with $E P H B 4$ variants was reported by a group focused primarily on vascular malformation syndromes other than HHT. ${ }^{2}$ Of other cases published to date, two cases were identified in dermatology clinics due to cutaneous $\mathrm{CMs},{ }^{20}$ and five others were ascertained due to vein of Galen malformations. ${ }^{3}$ This may explain the report of "only 


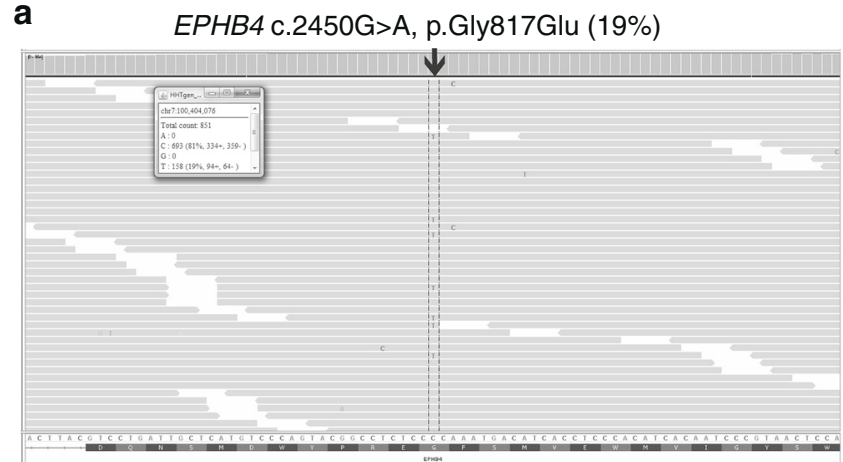

b

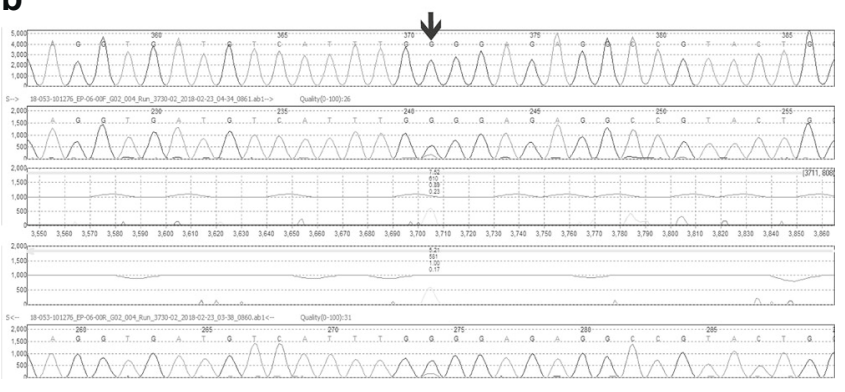

Fig. 2 EPHB4 mosaic variant causes capillary malformation--arteriovenous malformation 2 (CM-AVM2) in case 7. Next-generation sequencing (NGS) (a, 19\% variant) and Sanger sequencing data (b, 15\% variant) depict a mosaic EPHB4 variant in DNA extracted from blood.

a few" cases with epistaxis in the Amyere study; whereas 6 of the 10 cases in our series reported recurrent epistaxis. The ten cases presented here were ascertained at a medical center with a longstanding focus on the clinical and molecular diagnosis of HHT. And in our experience, a history of recurrent epistaxis, unless severe, is easy to miss without pointed questioning. However, given that at least some clinical suspicion of HHT existed in the majority of our cohort, the actual occurrence of epistaxis in patients with CM-AVM2 is likely to lie somewhere between that seen at our two centers.

Strikingly, all ten cases in our series had a pediatric presentation of symptoms that raised suspicion of HHT and/ or CM-AVM, and all were the first individual in their family diagnosed with a vascular malformation syndrome (Table 1). These patients had significantly more cutaneous vascular lesions than is typical for pediatric HHT patients (Fig. 3). The median number of cutaneous telangiectases in pediatric patients with HHT is two. It is uncommon for a child or adolescent with HHT to have more than a few telangiectases, and essentially unreported for one to have diffuse, innumerable telangiectases. ${ }^{21}$ Most commonly, HHT patients report the appearance of telangiectasia in the third decade. ${ }^{10}$ One recent study reported telangiectases in $16 \%$ of those $0-5$ years of age with HHT, and in $49 \%$ of those ages 16 to 21 (ref. ${ }^{22}$ ). Overwhelmingly, individuals diagnosed with HHT as a child or adolescent are not diagnosed based on their own clinical presentation, but by molecular genetic testing done based on family history.
Although oral and/or cutaneous telangiectases were reported in 8 of 10 cases in this series, no individual had telangiectases that typify those seen in HHT. In particular, the $1-2 \mathrm{~mm}$ pink to red punctate lesions that individually resemble those seen in HHT were notably different in terms of number and location on the body in these CM-AVM2 patients. It is not common in patients with HHT to have more than two dozen telangiectases at any age, and it is rare to have telangiectases "too numerous to count." ${ }^{21}$ Yet, this was common in this series of CM-AVM2 patients (Figs. 1 and 3). Of the five cases in this series in which telangiectases were reported and the number documented, three were said to have telangiectases "too numerous to count" or "diffuse/100s," and two cases were reported to have 27 and 30, respectively.

The reported locations of telangiectases overlap, but are not the same. Common sites in these CM-AVM2 cases were the extremities not limited to the hands, upper trunk, neck, face, and lips. In contrast, the oral cavity, hands/fingers, and lips are characteristic locations in HHT. Telangiectases on the trunk and extremities (other than hands) are not characteristic in HHT patients. ${ }^{9,10}$ While lips are a common site for telangiectases in both conditions, the large number of telangiectases on the lips, with involvement of the perioral area as in cases 3 (Fig. 1c), 6 (Fig. 1i), and 7 (Fig. 3a) in this series is not seen in HHT (Fig. 3b). All considered, the cutaneous vascular lesions are more widely and randomly distributed in CM-AVM2 than in HHT, and in this group of patients were notably more numerous than is typical in HHT.

Clinicians frequently use the word "telangiectasia" to describe both cutaneous lesions typical of HHT, as well as the small capillary malformations reported to characterize CM-AVM1 and CM-AVM2. The lack of uniformly accepted definitions of cutaneous vascular lesions has hampered the accurate characterization of vascular malformation syndromes. A telangiectasia is defined as a "dilated end blood vessel" and in the dermatological lexicon it is usually reserved for those vessels that can be visualized macroscopically, without the need for magnification. Capillary malformations are also dilated end vessels, which most typically occur in the superficial and mid dermis. The vessel caliber is too narrow to be visualized without magnification and appears as pink to red macules or patches. Both are descriptive terms and neither suggest underlying pathogenesis. The telangiectasia associated with HHT is somewhat of a misnomer. While they are dilated end vessels, representing loss of the microscopic capillary bed with dilation of postcapillary venules and subsequent shunting, the dilated vessels are not macroscopic. They would be more accurately defined as superficial dermal AVMs. Clinically, mature telangiectasias in HHT are red to purple in color, flat or slightly elevated, demonstrate fairly sharp borders, and are typically $1-4 \mathrm{~mm}$ in diameter. They demonstrate high flow with arterial high-pressure bleeding and arterial flow on Doppler interrogation. Many of the telangiectasias seen with CM-AVM2 appear to be single light red telangiectases with a surrounding blanched halo, suggestive of a vascular steal phenomenon. The other characteristic 

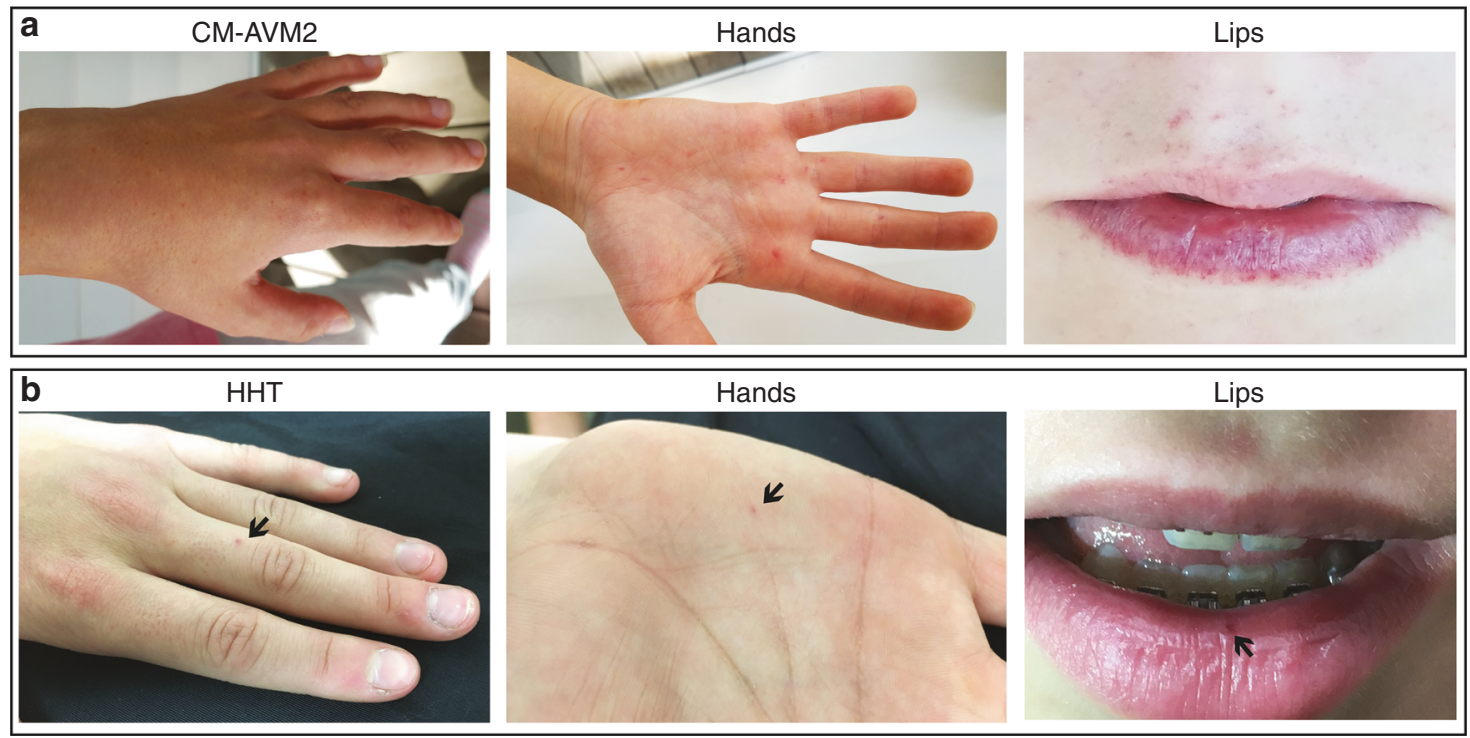

Fig. 3 Comparison of cutaneous telangiectases in capillary malformation--arteriovenous malformation 2 (CM-AVM2) versus hereditary hemorrhagic telangiectasia (HHT). In (a) and (b), dorsum and palmar images of the hand and lips are shown for a CM-AVM2 patient (case 7) and similaraged HHT patient, respectively. The few telangiectases in the HHT patient are indicated with an arrow.

capillary malformations observed in CM-AVM1 and 2 are pink, always flat macules and patches with variable blanched halos. They may have tiny macroscopic linear telangiectatic vessels as well. These also frequently demonstrate arterial flow and there is evidence in CM-AVM1 that deep dermal/ subcutaneous AVMs exist below these lesions. ${ }^{23}$

Nevertheless, patients in this series had a combination of the punctate telangiectases typical of HHT (Fig. 1k, o), spidery telangiectases that are less typical of HHT (Fig. 1p), and CMs previously described in CM-AVM1 and 2 (Fig. 1) but not in HHT. It is of note that the minority of the CMs in these patients were oval as often described in previous reports of CM-AVM. More typical in this series were CMs that were heterogeneous pink to red macules, without distinct borders, and of quite variable size. A fair number of the cutaneous vascular lesions in these patients might be described as either a large telangiectasia or small $\mathrm{CM}$, based on how these terms are typically used in HHT and CM-AVM (Fig. 1b, q).

The first case with an EPHB4 mosaic variant causing CMAVM2 was detected in this cohort. Interestingly, this case (case 7) was the most severely affected in this series in terms of distribution and number of telangiectases and CMs (Fig. 1 and Fig. 3a). Multiple sample types (blood, saliva, and buccal) from case 7 were found to carry the same EPHB4 variant at approximately the same level of mosaicism (19-25\%) indicating that the de novo variation occurred very early during development. Mosaicism has been reported previously in HHT, ${ }^{24}$ but never before in CM-AVM1 or 2. Therefore, it is important to evaluate cases suspected for CM-AVM using molecular genetic testing for EPHB4 that has the sensitivity to detect low levels of mosaicism.

Our cohort was heterogeneous in terms of the clinical evaluation and findings that led a clinician to suspect CM-
AVM and/or HHT. For this reason, it was not valid to calculate the chance of finding an EPHB4 variant in a patient who meets Curaçao criteria for the clinical diagnosis of HHT. In fact, it is of note that none of the individuals in this study in whom an EPHB4 variant was detected met consensus clinical criteria for HHT.

As a group, autosomal dominant conditions are known for variable phenotypic expression. It is likely that the phenotype of the recently described CM-AVM2 syndrome will continue to be expanded and refined as additional affected individuals are identified and carefully evaluated. Although CM-AVM2 clearly has some similarity to HHT, and overlaps in regard to cerebral AVM/AVF, the onset, pattern, and number of the cutaneous vascular lesions associated with each disorder are distinct. The presentation of multiple, if not numerous, telangiectases in pediatric patients was common in this group of CM-AVM2 patients, but is rare in pediatric HHT patients. At any age, diffuse, numerous telangiectases on the trunk, arms, or legs suggest CM-AVM, not HHT. Telangiectases (punctate, 1-2 mm) particularly if haloed and combined with "atypical small" $(0.5-4 \mathrm{~cm})$ CMs suggest CM-AVM, because CMs are not seen in HHT patients.

However, distinguishing the cutaneous lesions typical of CM-AVM versus HHT can present a challenge in the typical clinical setting. NGS panel testing of multiple genes included in the clinical differential will likely yield the highest clinical sensitivity. The recent discovery of a new CM-AVM gene, EPHB4, underscores the value of multigene panel testing for suspected CM-AVM syndrome. Our results demonstrate that patients who test negative for a variant in HHT genes may have a pathogenic variant in EPHB4. 


\section{ELECTRONIC SUPPLEMENTARY MATERIAL}

The online version of this article (https://doi.org/10.1038/s41436$019-0443-z)$ contains supplementary material, which is available to authorized users.

\section{ACKNOWLEDGEMENTS}

We thank the patients and their families for allowing us to publish their data. We thank members of the ARUP Molecular Genetics and Genomics Clinical Laboratories for assisting in the sequence analysis of these patients. We thank the ARUP Institute for Clinical and Experimental Pathology for funding this work. G.A. was supported by the Scientific and Technological Research Council of Turkey (TUBITAK) with a 2219-Postdoctoral Research Fellowship.

\section{DISCLOSURE}

The authors declare no conflicts of interest.

Publisher's note: Springer Nature remains neutral with regard to jurisdictional claims in published maps and institutional affiliations.

\section{REFERENCES}

1. Eerola I, Boon LM, Mulliken JB, et al. Capillary malformationarteriovenous malformation, a new clinical and genetic disorder caused by RASA1 mutations. Am J Hum Genet. 2003;3:1240-1249.

2. Amyere $M$, Revencu N, Helaers R, et al. Germline loss-of-function mutations in EPHB4 cause a second form of capillary malformationarteriovenous malformation (CM-AVM2) deregulating RAS-MAPK signaling. Circulation. 2017;136:1037-1048.

3. Vivanti A, Ozanne A, Grondin C, et al. Loss of function mutations in EPHB4 are responsible for vein of Galen aneurysmal malformation. Brain. 2018;141:979-988.

4. Boon LM, Mulliken JB, Vikkula M. RASA1: variable phenotype with capillary and arteriovenous malformations. Curr Opin Genet Dev. 2005; 15:265-269.

5. Revencu N, Boon LM, Mulliken JB, et al. Parkes Weber syndrome, vein of Galen aneurysmal malformation, and other fast-flow vascular anomalies are caused by RASA1 mutations. Hum Mutat. 2008;29:959-965.

6. Wooderchak-Donahue W, Stevenson DA, McDonald J, Grimmer JF, Gedge F, Bayrak-Toydemir P. RASA1 analysis: clinical and molecular findings in a series of consecutive cases. Eur J Med Genet. 2012;55:91-95.

7. Wooderchak-Donahue WL, Johnson P, McDonald J, et al. Expanding the clinical and molecular findings in RASA1 capillary malformationarteriovenous malformation (CM-AVM). Eur J Hum Genet. 2018;26:1521-1536.
8. McDonald J, Wooderchak-Donahue WL, VanSant Webb C, Whitehead K Stevenson DA, Bayrak-Toydemir P. Hereditary hemorrhagic telangiectasia: genetics and molecular diagnostics in a new era. Front Genet. 2015;6:1.

9. Shovlin C, Guttmacher $A E$, Buscarini $E$, et al. Diagnostic criteria for hereditary hemorrhagic telangiectasia (Rendu-Osler-Weber syndrome). Am J Med Genet. 2000;91:66-67.

10. Faughnan ME, Palda VA, Garcia-Tsao G, et al. International guidelines for the diagnosis and management of hereditary haemorrhagic telangiectasia. J Med Genet. 2011;48:73-87.

11. McDonald J, Bayrak-Toydemir P, Pyeritz R. Hereditary hemorrhagic telangiectasia: an overview of diagnosis, management, and pathogenesis. Genet Med. 2011;13:607-616.

12. Wooderchak-Donahue W, McDonald J, O'Fallon B, et al. BMP9 mutations cause a vascular-anomaly syndrome with phenotypic overlap with hereditary hemorrhagic telangiectasia. Am J Hum Genet. 2013;93:530-537.

13. Hernandez F, Huether R, Carter $L$, et al. Mutations in RASA1 and GDF2 identified in patients with clinical features of hereditary hemorrhagic telangiectasia. Hum Genome Var. 2015;2:15040.

14. Li H, Durbin R. Fast and accurate short read alignment with BurrowsWheeler transform. Bioinformatics. 2009;25:1754-1760.

15. Li H, Ruan J, Durbin R. Mapping short DNA sequencing reads and calling variants using mapping quality scores. Genome Res. 2008;18:1851-1858.

16. McKenna A, Hanna M, Banks E, et al. The Genome Analysis Toolkit: a MapReduce framework for analyzing next-generation DNA sequencing data. Genome Res. 2010;20:1297-1303.

17. Ng PC, Henikoff S. SIFT: predicting amino acid changes that affect protein function. Nucleic Acids Res. 2003;31:3812-3814.

18. Schwarz JM, Rodelsperger C, Schuelke M, Seelow D. MutationTaster evaluates disease-causing potential of sequence alterations. Nat Methods. 2010:7:575-576.

19. Adzhubei IA, Schmidt S, Peshkin L, et al. A method and server for predicting damaging missense mutations. Nat Methods. 2010;7:248-249.

20. Yu J, Streicher JL, Medne L, Krantz ID, Yan AC. EPHB4 mutation implicated in capillary-malformation-arteriovenous malformation syndrome: A case report. Pediatr Dermatol. 2017;34:e227-e230.

21. Gonzalez CD, Mcdonald J, Stevenson DA, Whitehead KJ, Petersen MG, Presson AP, Ding Q, Wilson KF. Epistaxis in children and adolescents with hereditary hemorrhagic telangiectasia. Laryngoscope, 2018;128: 1714-1719.

22. Pahl KS, Choudhury A, Wusik K, et al. Applicability of the Curacao criteria for the diagnosis of hereditary hemorrhagic telangiectasia in the pediatric population. J Pediatr. 2018;197:2017-2213.

23. Kim C, Ko CJ, Baker KE, Antaya RD. Histopathologic and ultrasound characteristics of cutaneous capillary malformations in a patient with capillary malformation-arteriovenous malformation syndrome. Pediatr Dermatol. 2015;32:128-131

24. Best DH, Vaughn C, McDonald J, et al. Mosaic ACVRL1 and ENG mutations in hereditary haemorrhagic telangiectasia patients. J Med Genet. 2011;48:358-360. 